\title{
EMAP-DCNN: A NOVEL MATHEMATICAL MORPHOLOGY AND DEEP LEARNING COMBINED FRAMEWORK FOR HYPERSPECTRAL IMAGE CLASSIFICATION
}

\author{
H. Teffahi ${ }^{1 *}$, N. Teffahi ${ }^{2}$ \\ ${ }^{1}$ ASAL, Algerian Space Agency, Space Application Center (CAS), Bouzareah, Algiers - hteffahi@asal.dz \\ (teffahi.hanane89@gmail.com) \\ ${ }^{2}$ National Superior School of Marine Science and Coastal Planning, Dely Ibrahim, Algiers - teffahin@ yahoo.fr
}

\begin{abstract}
KEY WORDS: Extended Multi-Attribute Profiles (EMAP), Dense Conventional Neural Network (DCNN), Spectral-spatial Classification, Deep Learning (DL), Mathematical Morphology, Feature Extraction and Fusion, Hyperspectral Remote Sensing Image classification.
\end{abstract}

\begin{abstract}
:
The classification of hyperspectral image (HSI) with high spectral and spatial resolution represents an important and challenging task in image processing and remote sensing (RS) domains due to the problem of computational complexity and big dimensionality of the remote sensing images. The spatial and spectral pixel characteristics have crucial significance for hyperspectral image classification and to take into account these two types of characteristics, various classification and feature extraction methods have been developed to improve spectral-spatial classification of remote sensing images for thematic mapping purposes such as agricultural mapping, urban mapping, emergency mapping in case of natural disasters... In recent years, mathematical morphology and deep learning (DL) have been recognized as prominent feature extraction techniques that led to remarkable spectral-spatial classification performances. Among them, Extended Multi-Attribute Profiles (EMAP) and Dense Convolutional Neural Network (DCNN) are considered as robust and powerful approaches such as the work in this paper is based on these two techniques for the feature extraction stage and used in two combined manners and constructing the EMAP-DCNN frame. The experiments were conducted on two popular datasets: "Indian Pines" and "Huston" hyperspectral datasets. Experimental results demonstrate that the two proposed approaches of the EMAP-DCNN frame denoted EMAP-DCNN 1, EMAP-DCNN 2 provide competitive performances compared with some state-of-the-art spectralspatial classification methods based on deep learning.
\end{abstract}

\section{INTRODUCTION}

In the last decades, with the rapid development of technology Remote Sensing data has become an essential source of spatial and land cover information. The various remote sensing images are applied to solve many problems in different fields as environment, urban planning, Agriculture, due to the very high spatial and spectral resolutions. But as this point is an advantage, it also gives rise to a limitation because it increases the correlation between adjacent bands and brings out a large amount of information redundancy [1], [2].

To overcome these limitations, with the aim to improve the classification accuracy of remote sensing images, feature extraction, selection, and fusion techniques became an important tool for the classification process specifically for hyperspectral image classification.

To achieve a powerful pixel-wise classification of hyperspectral imagery, different methods were developed. The first methods of HSI classification have focused on the utilization of the spectral signatures of HSIs for the classification task (e.g., neural networks [3], support vector machines (SVM) [4], logistic regression [5],[6]. In addition, some other classification approaches have based on effective feature extraction or dimension reduction techniques, such as principle component analysis (PCA) [7] [8], independent component analysis (ICA) [9], [10], however, the classification results obtained by these classifiers are not good enough since the spatial information isn't taken into account.

In recent years, spatial features have been demonstrated to be very useful in improving the representation of hyperspectral data and increasing the classification accuracies [11], [12]. In this context, many spectral-spatial classification frameworks have been proposed. According to the latest research works, the spatial information was added to the spectral features by applying spatial feature extraction techniques such as grey-level co-occurrence matrix (GLCM), Markov random field (MRF) [13], [14], morphological profiles (MP) [15], attributes profiles (AP) [16], extinction profiles (EP) [17],...etc.

In the last decay, Deep Learning has become a growing trend in big data analysis and great breakthrough has been made with the approach in many computer vision tasks, e.g., image classification [18], [19], object detection [20], and natural language processing [21]. The deep learning approaches have attracted much attention for their potential as feature extraction, selection, and fusion techniques to classify HIS images and achieve good performance. In 2014, Yushi Chen first proposed a deep learning framework to merge spatial and spectral features [22]. The deep learning framework (SAE-LR) combined PCA with deep learning architecture then used Stacked Autoencoders (SAE) to obtain high-level features and fed into logistic regression classifier. SAE-LR had a large potential for HSI classification but it has a disadvantage in terms of its training

\footnotetext{
* Corresponding author
} 
time. In the following year, Makantasis in [23] used a convolutional neural network $(\mathrm{CNN})$, a deep supervised method for HSI classification. Recently, CNN has achieved big success in the remote sensing image processing field. In 2017, Gao et al. proposed a new variant of CNN, DenseNet [24] considered as a deep network structure based on Residual Net (ResNet) [25] and Google Inception [26]. DenseNet represents a more powerful version of ResNet, which can reduce the problem of gradients becoming zero such as the structure can effectively use features and enhancing their transfer between convolution layers. However, CNN has a disadvantage point that is the inaccurate boundary location problem, which leads to obtaining incomplete object shapes. Therefore, the representation capability of features from deep networks can be complemented with information captured with another type of spatial extractor [27].

In this paper, we investigate the classification of hyperspectral images based on the EMAP-DCNN framework, which is constructed by Extended Multi-Attribute Profiles (EMAP) for extracting spatial features and Dense-CNN (DCNN) for extraction high-level features and dimensionality reduction. The structure of the EMAP-DCNN framework is done in two manners, in the first version of the feature extraction framework (EMAP-DCNN-1), Extended Multi-Attribute Profiles (EMAP) was first performed. Then, a Dense Convolutional Neural Network (DCNN) was applied to extract high-level features from the EMAP output vector. Finally, the dense fully connected layer is fed into SVM or Softmax classifier. In the second version (EMAP-DCNN-2), EMAP spatial extractor was first performed and stacked with the original spectral features. Then, DCNN was applied to extract top spectral-spatial features. In the last step SVM or Softmax classifier was used for performing the classification process.

\section{PROPOSED METHODOLOGY}

In this section, we first provide an overview of the EMAPDCNN framework shows in Figure 1 (sub-section 2.1). The proposed methodology is presented on two different architectures to define feature extraction techniques which serve to perform a supervised classification.

\section{EMAP-DCNN 1}
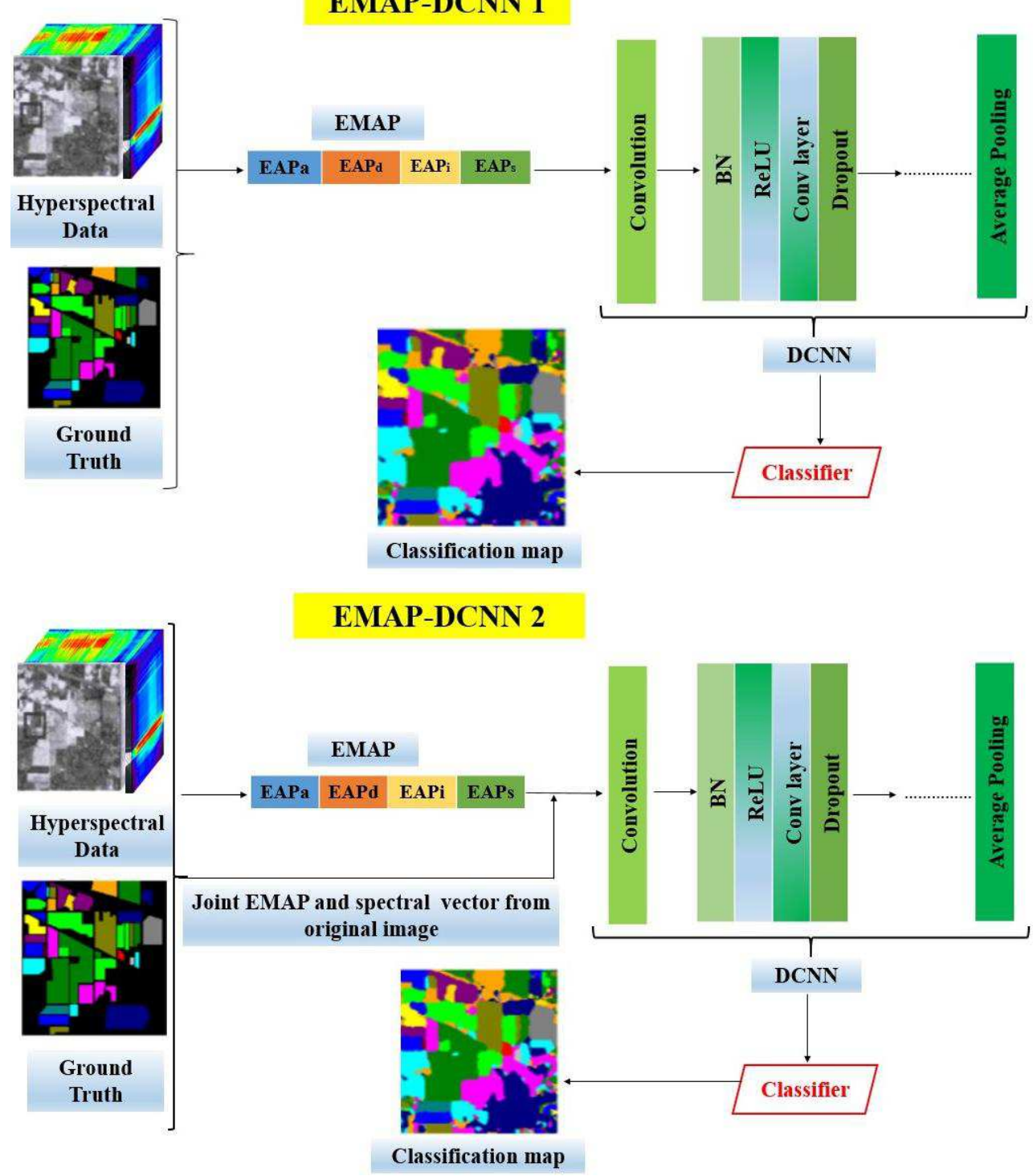

Figure 1. The General process of EMAP-DCNN Frame with the two approaches. 
Our methodology is proposed in two approaches EMAP-DCNN1 and EMAP-DCNN-2. The extended multi-attribute profiles (EMAP) are used to extract the spatial features from the hyperspectral data and The Dense-CNN with batch normalization, rectified LU (ReLU) and dropout is developed to extract abstract and robust features. At the end of the framework, softmax or SVM classifiers are employed to produce the final classification map. Details of the proposed framework EMAPDCNN are discussed in sub-Section 2.2.

\subsection{Extended Multi-Attribute Profiles (EMAP)}

Extended Multi-Attribute Profiles (EMAP) are constructed by the concatenation of different Extended Attribute Profiles (EAP). EMAP has demonstrated a greater capability to extract the spatial information from the remote sensing images than a single EAP [13] such as EMAP is computed from remote sensing datasets with various attributes: $a$ : area of the regions, $d$ : length of the diagonal of the box bounding the region, $i$ : a moment of inertia and $s$ : standard deviation of the gray-level values of the pixels in the regions. The attributes $a$ and $d$ represent respectively "the area of the regions" and "the length of the diagonal of the box bounding the region", are growing attributes that are efficient and useful to realize a multilevel data analysis. On the other hand, the attribute $i$ "moment of inertia" is a geometric attribute that measures the elongation of the regions and the attribute $s$ "standard deviation" measures the homogeneity of the intensity values of the pixels belonging to each region in the image and brings information dependent on the spectral contrast of the pixels [16], [28].

Dalla Mura introduced Attribute Profiles (AP). APs present a multilevel characterization structure of an image $f$ applying successively morphological attribute filters [16], [29]. Attribute Profiles were defined as the successive application of thinning and thickening transformations. This process is considered as a generalization of the opening and closing process employed for Morphological Profiles (MP) such that the reconstruction is implemented as attribute filters.

Attribute filters operate on the connected components (i.e., regions of spatially connected pixels) that compose an image, according to a specific criterion evaluated on each connected component $\mathrm{CC}$ of the image $f$ and associated with thickening and thinning transformations. However, the AP is defined as computing an attribute A for every connected component $\mathrm{CC}$ of an image $f$ for a given reference value $\lambda$. For each connected component $\mathrm{C}_{i}$ of the image, whether the attribute satisfies a predefined criterion, afterward the region remains unaffected; otherwise, it is set to the radiometric value of the adjacent region with the nearest value, so $C_{i}$ can be merged to the adjacent connected component. When the region is merged to the adjacent region of a lower (or higher) grey level, the process achieved is a thinning (or a thickening) [28].

An AP is a successive chain of attribute thinning and attribute thickening with considering a sequence of thresholds $\left\{\lambda_{1}, \lambda_{2} \ldots\right.$. ,$\left.\lambda_{n}\right\}$ as follows:

$$
\operatorname{AP}(f)=\left\{\phi_{n}(f), \ldots, \phi_{1}(f), f, \gamma_{1}(f), \ldots, \gamma_{n}(f)\right\}
$$

Such as $\varphi_{i}$ and $\gamma_{i}$ denote respectively the thickening and thinning transformations, with $n$ attributes thickening $\left(\varphi^{\mathrm{T}}\right)$ and $n$ attributes thinning $\left(\gamma^{\mathrm{T}}\right)$. Therefore, each pixel $p$ of an image $f$ can be configured and typified using the values resulting from the successive filtering operations.
The concept of AP was extended to multispectral and hyperspectral images with the definition of Extended Attribute Profiles (EAP) where an EAP is computed by concatenating the APs considering the same attribute of the $r$ principal components $\mathrm{PCs}$, extracted from the original image [16]:

$$
\mathrm{EAP}=\left\{\mathrm{AP}\left(\mathrm{PC}_{1}\right), \mathrm{AP}\left(\mathrm{PC}_{2}\right), \ldots, \mathrm{AP}\left(\mathrm{PC}_{\mathrm{r}}\right)\right\}
$$

Where $\mathrm{PC} i(1 \leq i \leq r)$ are the $r^{\text {th }}$ first principal components obtained after applying PCA for the original hyperspectral image. PCA uses performing attribute filtering on the $r^{\text {th }}$ first PCs in order to reduce computational complexity.

For conclusion, EMAP presents the concatenation of the EAPs $\left(\mathrm{EAP}_{a}, \mathrm{EAP}_{d}, \mathrm{EAP}_{i}, \mathrm{EAP}_{s}\right)$ :

$$
\operatorname{EMAP}=\left[\operatorname{EAP}_{a}, \operatorname{EAP}_{d}, \operatorname{EAP}_{i}, \mathrm{EAP}_{s}\right]
$$

\subsection{Dense Convolutional Neural Network (DCNN)}

Convolutional Neural Network (CNN) is a supervised deep learning method that presented a multilayer model that can be trained from a remote sensing image to the final classification map. The feature extraction (FE) system based on a typical CNN model consists of convolutional layers, activation layers, pooling layers, fully connected layer, and a classification layer.

Dense Convolutional Network (DenseNet) considered as a new deep structure of CNN which represents a more powerful version of ResNet and can be used to exploit the potential of the network through feature reuse, yielding condensed models that are easy to train and highly parameter efficient [30]. To further enhance the information for throughout a traditional convolutional network, Huang and his collaborators [13] proposed a new network, called DenseNet (DCNN), such as in this network shortcut connections are employed to concatenate the input features with the output features instead of adding while others $\mathrm{CNN}$ architectures with $\mathrm{L}$ layers have L connections (one between each layer and its subsequent layer).

Suppose that the CNN has L convolution layers, $X_{l}$ is the output of the $l^{\text {th }}$ layer and $H_{l}()$ represents the complex nonlinear transformation operations in the $l^{\text {th }}$ convolution layer. In the connected structure of the traditional CNN, the output of the ( $l$ $1)^{\text {th }}$ layer is the input of the $l^{\text {th }}$ layer as follow :

$$
X_{l}=H_{l}\left(X_{l-1}\right), \quad l \in N^{+}
$$

In the $\mathrm{CNN}$ architectures, pooling layers increase the robustness of the learned features, change the spatial size of feature maps, resulting in the concatenation operation being unfeasible. To address this problem, Huang et al. [24] divided the network into multiple dense blocks, which do the dense connections in each block and add a pooling layer behind each block [31], as shown in Figure 2 and Figure 3.

DenseNet connects each layer to every other layer in a feed forward manner, it means that DCNN acquires feature maps from all preceding layers as input for the next layer. DCNN uses an extremely densely-connected structure, with the feature map of the output of the zeroth to the $(l-1)^{\text {th }}$ layers acting as the input to the lth layer. The connected structure is formulated as:

$$
X_{l}=H_{l}\left[X_{0}, X_{1}, \ldots, X_{l-1}\right], \quad l \in N^{+}
$$




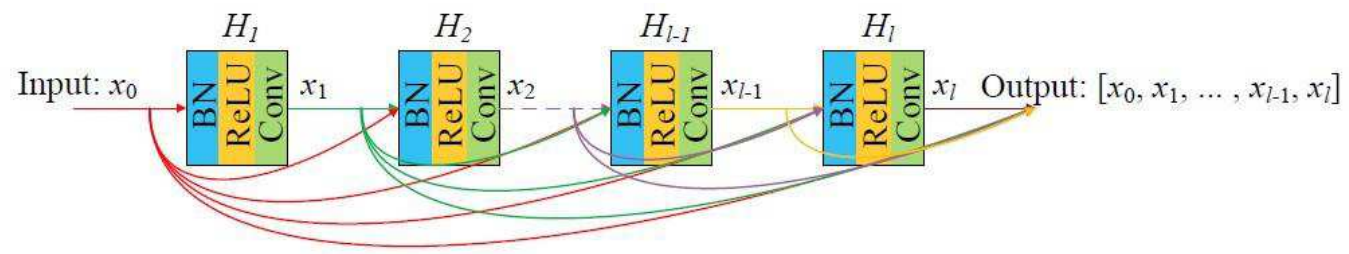

Figure 2. The general structure of a dense block [32]: BN-ReLu-Conv.

Where [.] denotes the concatenation operator and $H_{l}()$ is a composite conventional layer (Conv layer) with the structure of BN-ReLU-Conv [32] constructed by: Batch Normalization (BN), Restricted LU (ReLU) and Conventional layer (Conv). Finally, input features and those generated by each Conv layer are concatenated as the output of the dense block, as shown in Figure 3.
In DCNN, only layers within each block are densely connected and constructed a local dense connectivity pattern (Figure 3). After each dense block (not the last one), convolutional layers are utilized for more compacting the extracted features, however, the non-dense connections between each block conduct the network to focus more on the high level features extracted by the last dense block for image classification.

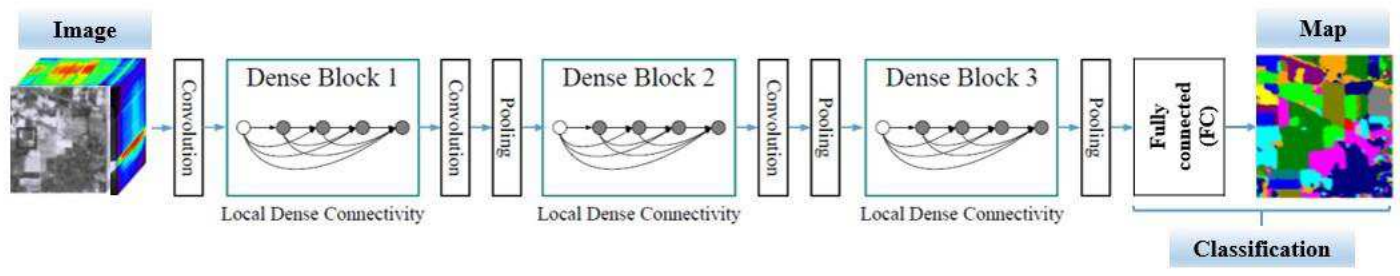

Figure 3. General flowchart of image classification based on DenseNet [24]

In our paper, $H_{l}()$ is the composite function defined as four successive operations: batch normalization (BN) [33], ReLU, convolution a,nd dropout [29] as it's shown in Figure 1.

In the first step of DCNN for our methodology, a convolution is constructed from the EMAP features as the input of the EMAPDCNN 1 and input of joint EMAP features with the original hyperspectral image. Let $N F^{l}$ the number of filters in layer $l$ and $X_{i}^{l}$ is the $i^{\text {th }}$ input feature tensor; * is the convolution operator; $H_{j}^{l+1}$ and $b_{j}^{l+1}$ are the $j^{\text {th }}$ filter and bias in layer $l+1$, respectively, where the output $X_{j}^{l+1}$ of the convolution operation is defined as follow:

$$
X_{j}^{l+1}=\sum_{i=1}^{l} X_{i}^{l} * H_{j}^{l+1}+b_{j}^{l+1} \quad l \in N^{+}
$$

The filter size in this layer is set to $1 \times 1 \times \mathrm{N}$ ( $\mathrm{N}$ is the number of bands) and the $1 \times 1$ spatial size is set to exploit the EMAP features.

Batch normalization used for constructing dense block is usually applied to a convolutional network before the activation function. It has the following main effects: 1) it helps to obtain faster learning since the learning rate can be increased compared to the non-batch-normalized version and 2) flexibility on mean and variance values for each dimension in every layer which increases the accuracy of the network [29].

$\mathrm{BN}$ is illustrated as [33]:

$$
\tilde{X}^{l}=\frac{X^{l}-E\left(X^{l}\right)}{\operatorname{VAR}\left(X^{l}\right)} \quad l \in N^{+}
$$

Where $X^{l}$ denotes the $l^{\text {th }}$ layer's batch feature maps, $E\left(X^{l}\right)$ is the expectation of $X^{l}$, Similarly, $\operatorname{VAR}\left(X^{l}\right)$ is the variance of $X^{l}$. The output $\tilde{X}^{l}$ is the normalization result of the input tensor. The $\mathrm{BN}$ results are convolved with $1 \times 1$ or $3 \times 3$ where $\mathrm{BN}$ is followed by a rectified linear unit [30] ReLU as the activation function. The ReLU function is defined as:

$$
\hat{Z}=\max \{0, Z\}
$$

Such as $\mathrm{Z}$ is the input tensor.

To further address the overfitting problem, we add a dropout operation after ReLU such as dropout sets the output generated by some neurons to zero with a specific probability.

After the last dense block of the framework, an average pooling layer is used. The average of the output feature maps is calculated in this layer. The global average pooling layer contains a much smaller number of parameters than fully connected (FC) layers and can retain localization ability for a network. After the FC layer, a softmax layer or SVM is used to obtain the final classification result.

It is efficient to consider two main problems in HIS classification: the over-fitting phenomenon caused by the large model scale with limited training data and the effective extraction for both spectral and spatial features. Dense networks (DCNN) and EMAP have several compelling advantages to alleviate the vanishing-gradient problem, strengthen feature propagation, encourage feature reuse, and reduce the number of parameters.

\section{EXPERIMENTS RESULTS AND DISCUSSION}

In this section, we provide a detailed description of the Datasets, discuss the results of the experiment and validate the performance of the EMAP-DCNN framework.

Two publicly hyperspectral datasets were utilized to verify the effectiveness of our EMAP-DCNN method, i.e., Indian Pines (IP) and the University of Houston (UH). 
The IP dataset was gathered in 1992 by the Airborne Visible/Infrared Imaging Spectrometer (AVIRIS) sensor. The dataset covers an agricultural area in Northwest Indiana (Figure 4). IP image consisting of $145 \times 145$ pixels and 224 spectral bands in the spectral range from 400 to $2500 \mathrm{~nm}$. The spatial resolution is $20 \mathrm{~m}$, and the ground reference map has 16 classes (Table 1).

The Huston dataset (UH) was acquired by the compact airborne spectrographic imager over the University of Houston campus and the neighbouring urban area on June 23, 2012, and was distributed for the 2013 GRSS data fusion contest. The size of the data is $349 \times 1905$ pixels with a spatial resolution of $2.5 \mathrm{~m}$ and consists of 144 spectral bands ranging from 0.38 to $1.05 \mu \mathrm{m}$. The ground truth map contains 15 classes of interests. Figure 5 shows a color composite representation of the UH data and the corresponding ground truth data. Table 2 gives information about the number of total samples for different classes of interest.

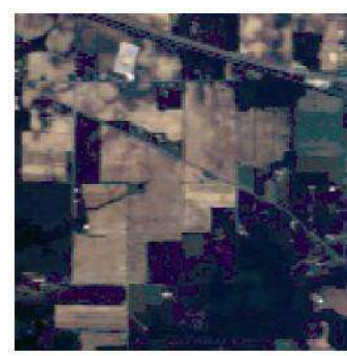

(a) False color image

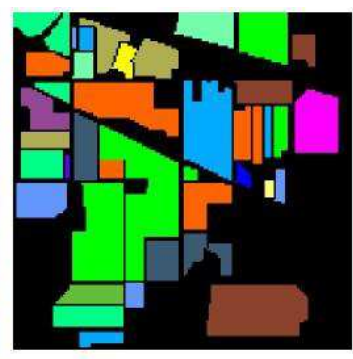

(b) Ground reference map

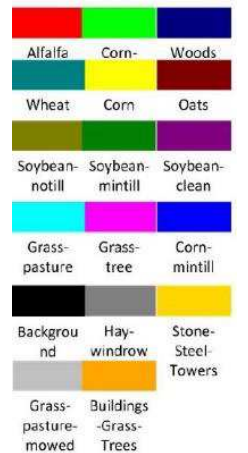

Figure 4. Indian Pines data description
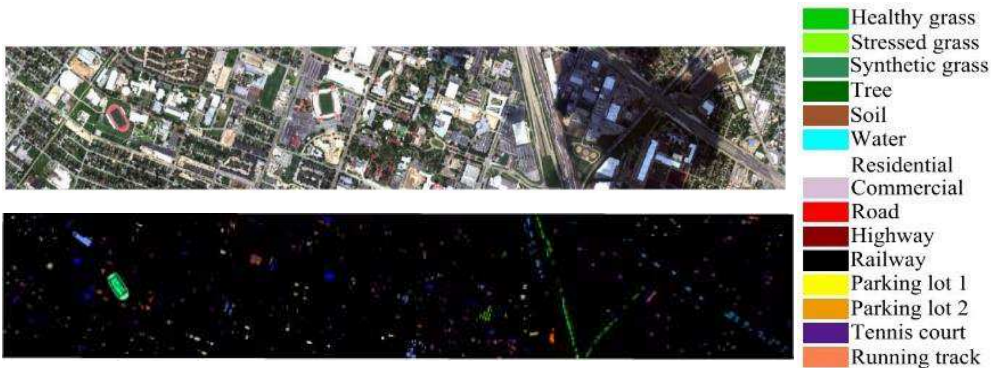

Figure 5. Huston data description: (Up) hyperspectral Huston image (Down) Ground truth data.

Started by computing EMAP extractor, four attributes [(1) area of the regions (a); (2) diagonal of the box bounding the region $(d)$; (3) moment of inertia $(i)$; and (4) standard deviation of the gray-level values of the pixels in the regions $(s)]$ [16].

For the first hyperspectral data "Indian Pines" (IP), each EAP leads to 36_dimentional profiles (composed by four Aps of nine levels computed on the $4 \mathrm{PCs}$ ). The $\lambda$ values (threshold value)

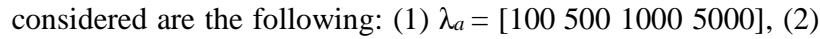
$\lambda_{d}=\left[\begin{array}{llll}10 & 25 & 50 & 100\end{array}\right]$, (3) $\lambda_{i}=\left[\begin{array}{llll}0.2 & 0.3 & 0.4 & 0.5\end{array}\right]$, (4) $\lambda_{s}=\left[\begin{array}{lll}20 & 30 & 40\end{array}\right.$ 50].

Then, for the second hyperspectral data "Huston data", each EAP leads to 44 -dimensional profiles. The $\lambda$ values considered are the

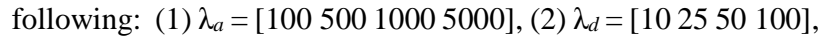
(3) $\lambda_{i}=\left[\begin{array}{llll}0.1 & 0.15 & 0.2 & 0.25\end{array}\right]$, (4) $\lambda_{s}=\left[\begin{array}{llll}15 & 20 & 25 & 30\end{array}\right]$.

Due to the high dimensionality of the generated attributes. We use DCNN as a feature extraction technique for extracting highlevel features and dimensionality reduction.
Table 1. Ground reference classes of Indian Pines (IP)

\begin{tabular}{ccc}
\hline Class number & Class name & Total Samples \\
\hline 1 & Alfalfa & 46 \\
2 & Corn-notill & 1428 \\
3 & Corn-mintill & 830 \\
4 & Corn & 237 \\
5 & Grass-pasture & 483 \\
6 & Grass-trees & 730 \\
7 & Grass-pasture-mowed & 28 \\
8 & Hay-windrowed & 478 \\
9 & Oats & 20 \\
10 & Soybean-notill & 972 \\
11 & Soybean -mintill & 2455 \\
12 & Soybean-clean & 593 \\
13 & Wheat & 205 \\
14 & Woods & 1265 \\
15 & Building-grass-trees-drives & 386 \\
16 & Stone-steal-towers & 93 \\
\hline
\end{tabular}


Table 2. Ground reference classes of Huston data (UH)

\begin{tabular}{ccc}
\hline Class number & Class name & Total Samples \\
\hline 1 & Grass healthy & 1251 \\
2 & Grass stressed & 1254 \\
3 & Grass & 697 \\
4 & synthetic & 1244 \\
5 & Tree & 1242 \\
6 & Soil & 325 \\
7 & Water & 1268 \\
8 & Residential & 1244 \\
9 & Commercial & 1252 \\
10 & Road & 1227 \\
11 & Highway & 1235 \\
12 & Railway & 1233 \\
13 & Parking lot 1 & 469 \\
14 & Parking lot 2 & 428 \\
15 & Tennis court & 660 \\
\hline
\end{tabular}

We configured our DCNN as follows: We tested our network with patch sizes $\{5 \times 5 ; 7 \times 7 ; 9 \times 9 ; 11 \times 11\}$ and found that the patch size of $7 \times 7$ for IP data and $9 \times 9$ for UH data were the most suitable to yield satisfactory performance.

The DCNN used in our method is composed of two dense blocks, after the first step of convolution with $1 \times 1 \times \mathrm{N}$ where $3 \times 3$ filters are adopted to exploit spectral-spatial features in each dense block. The $3 \times 3$ convolution operation can address the spatial correlation of neighbouring pixels.

In the training process, with the batch size of 32, where the initial learning rate was set to 0.0001 for IP data and 0.001 for UH data (for EMAP-DCNN1) and 0.001 for IP data and 0.01 for UH data (for EMAP-DCNN2). The best precision was reached within 40 epochs represented the number of epochs used during the training stage for Indian Pines data and 80 epochs for Huston data (for EMAP-DCNN1), adding we used 80 epochs for IP data and 120 epochs for UH data (for EMAP-DCNN2). In addition, the dropout rate is set to 0.5 . The average pooling layer is used with the kernel size 3 and the stride 2. The softmax classifier was retained for the final step of classification because of its better results than the SVM.

All the training and testing results of the two approaches of the EMAP-DCNN framework were obtained on the same computer, with the configuration of 32 GB of memory, NVIDIA GeForce GTX 1060 3GB and Intel i7. The experiments are also tried on GPU configured on Google Colab with only $12 \mathrm{~GB}$ of memory.

In our experiments, overall accuracy (OA \%), average accuracy (AA\%), and the Kappa coefficient $(\mathrm{K} \times 100)$ were chosen to evaluate the classification performance of the methods. To provide a statistical evaluation, each experiment was repeated 10 times, and the mean was reported. The results of the experiments are shown in Tables 3, 4, and in Figures 6, 7.
Table 3. Classification accuracies of EMAP-DCNN 1.

\begin{tabular}{cccc}
\hline Data & OA & Kappa & AA \\
\hline Indian Pines & 99.75 & 99.53 & 99.49 \\
Huston & 99.62 & 99.54 & 99.47 \\
\hline
\end{tabular}

Table 4. Classification accuracies of EMAP-DCNN 2.

\begin{tabular}{cccc}
\hline Data & OA & Kappa & AA \\
\hline Indian Pines & 99.86 & 99.74 & 99.69 \\
Huston & 99.7 & 99.66 & 99.59
\end{tabular}

EMAP-DCNN Frame is compared with different frameworks applied to the same datasets. In our experiment, we compared the proposed EMAP-DCNN framework to other deep-learningbased methods (Table 5), that are, (M1) SAE-LR [22], (M2) CNN [24], (M3) 3D-CNN-LR [25], (M4) SSDC-DenseNet [34]. These methods were programmed by the authors during a long time of research work. The proposed approaches of EMAPDCNN are denoted MP1 and MP2.

Table 5. Comparison of the overall classification accuracies

\begin{tabular}{ccccccc}
\hline Data & M1 & M2 & M3 & M4 & MP1 & MP2 \\
\hline IP & 95.30 & 95.96 & 97.20 & 99.53 & $\mathbf{9 9 . 7 5}$ & $\mathbf{9 9 . 8 6}$ \\
UH & 93.80 & 90.83 & 96.77 & 98.96 & $\mathbf{9 9 . 6 2}$ & $\mathbf{9 9 . 7}$
\end{tabular}

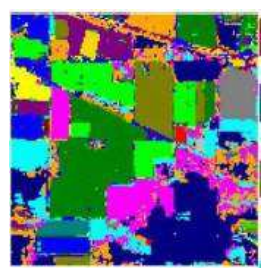

(M1)

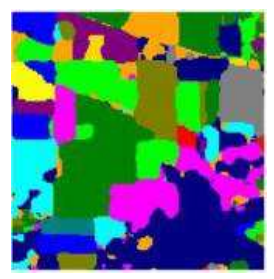

(M4)

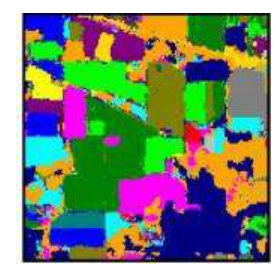

(M2)

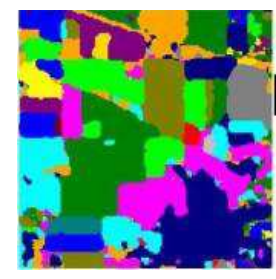

(MP1)

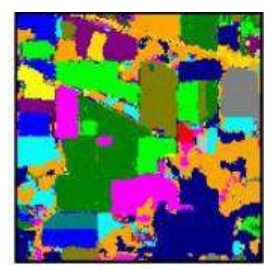

(M3)

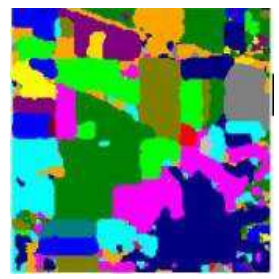

(MP2)
Figure 6. Classification maps for Indian Pines data.

The objective of our work is to propose a new spectral-spatial framework based on mathematical morphology and deep learning model in order to increase the classification accuracies and robust classification maps with high level extracted features. We attend a high classification accuracy but still have a big running time. 


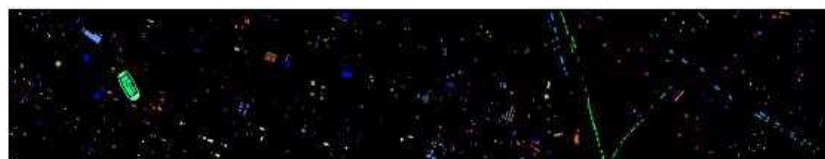

(M1)

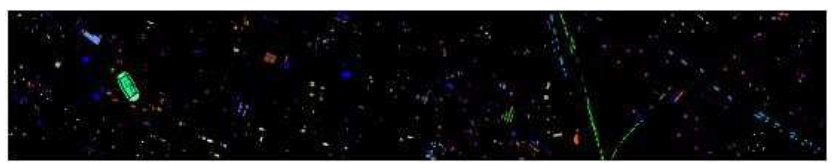

(M2)

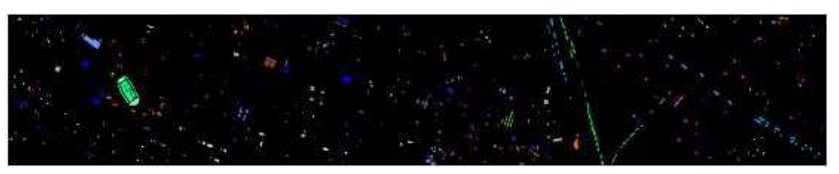

(M3)

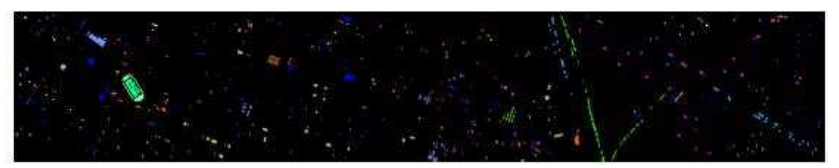

(M4)

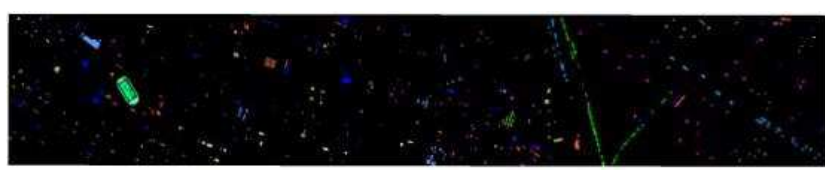

(MP1)

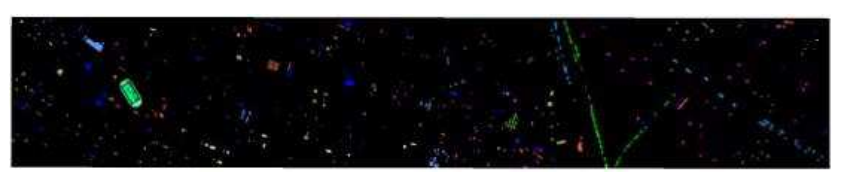

(MP2)

Figure 7. Classification maps for Huston data.

\section{CONCLUSION}

In this letter, a new framework based on mathematical morphology and deep learning feature extractors for the hyperspectral data classification was proposed. The welldesigned DCNN was used to extract the discriminative features that led to good classification performance.

The EMAP-DCNN with the two approaches EMAP-DCNN1 and EMAP-DCNN2 achieved $99.86 \%$ and $99.7 \%$ in terms of OA on Indian Pines and Houston datasets, respectively, when training rate is reduced from 0.01 until 0.0001 .

In order to boost the classification accuracy, Extended MultiAttribute Profiles was combined with Dense-CNN and outperform a simple DCNN in terms of OA, AA, and Kappa coefficient.

The results obtained by the proposed approaches of EMAPDCNN Frame on the two hyperspectral datasets demonstrated that the new deep CNN models have huge potential for accurate hyperspectral data classification and consequently EMAPDCNN Frame will also be applied for multispectral, UAV and LIDAR image classification as our future work and we will try to outperform the running times of our developed models, the work will also extend by using novel deep learning methods as Capsule-network.

\section{REFERENCES}

[1] Akar, Ö., 2017. Mapping land use using Rotation Forest algorithm from UAV images. European Journal of Remote Sensing 50, pp. 269-279.

[2] Teffahi, H., Yao, H., Belabid, N., Chaib, S., 2018. Feature Extraction based on Extend Multi-Attribute Profiles and Sparse Autoencoder for Remote Sensing Image Classification. Proceedings of SPIE conference on Multispectral Image Acquisition, Processing, and Analysis (MIPPR), 10607.

[3] Zhong Y., and Zhang, L., 2012. An adaptive artificial immune network for supervised classification of multi-/hyperspectral remote sensing imagery. IEEE Transactions on Geoscience and Remote Sensing 50(3), pp. 894-909.

[4] Melgani F., and Bruzzone, L., 2004. Classification of hyperspectral remote sensing images with support vector machines. IEEE Transactions on Geoscience and Remote Sensing 42(8), pp. 1778-1790.

[5] Li, J., Bioucas-Dias, J. M., and Plaza, A., 2010. Semisupervised hyperspectral image segmentation using multinomial logistic regression with active learning. IEEE Transactions on Geoscience and Remote Sensing 48(11), pp. 4085-4098.

[6] Li, J., Bioucas-Dias, J. M., and Plaza, A., 2012. Spectralspatial hyperspectral image segmentation using subspace multinomial logistic regression and markov random fields. IEEE Transactions on Geoscience and Remote Sensing 50(3), pp. 809823.

[7] Agarwal, A., El-Ghazawi, T., El-Askary, H., Le-Moigne, J., 2007. Efficient hierarchical-PCA dimension reduction for hyperspectral imagery. In: Proceedings of the IEEE international symposium on signal processing and information technology. Giza, Egypt, pp. 353-356

[8] Licciardi, G., Marpu, P. R., Chanussot, J., and Benediktsson, J. A., 2012. Linear versus nonlinear PCA for the classification of hyperspectral data based on the extended morphological profiles. IEEE Geosciences and Remote Sensing Letters 9(3), pp. 447451.

[9] Falco, N., Benediktsson, J. A., Bruzzone, L., 2015. Spectral and Spatial Classification of Hyperspectral Images Based on ICA and Reduced Morphological Attribute Profiles. IEEE Transactions on Geoscience and Remote Sensing 53(11), pp. $6223-6240$.

[10] Villa, A., Benediktsson, J. A., Chanussot, J., and Jutten, C., 2011. Hyperspectral image classification with independent component discriminant analysis. IEEE Transactions on Geoscience and Remote Sensing 49(12), pp. 4865-4876.

[11] Ghamisi, P., et al., 2018. New frontiers in spectral-spatial hyperspectral image classification: The latest advances based on mathematical morphology, markov random fields, segmentation, sparse representation, and deep learning. IEEE Geoscience and Remote Sensing Magazine 6(3), pp. 10-43.

[12] He, L., Li, J., Liu, C., and Li, S., 2018. Recent advances on spectral-spatial hyperspectral image classification: An overview 
and new guidelines. IEEE Transactions on Geoscience and Remote Sensing 56(3), pp. 1579-1597.

[13] Ghamisi, P and Benediktsson, J.A., 2014. Automatic Framework for Spectral-Spatial Classification Based on Supervised Feature Extraction and Morphological Attribute Profiles. IEEE Journal of Selected Topics in Applied Earth Observations and Remote Sensing 7(6), pp. 2147-2160.

[14] Bhardwaj, K., and Patra, S., 2018. An unsupervised technique for optimal feature selection in attribute profiles for spectral-spatial classification of hyperspectral images. ISPRS Journal of Photogrammetry and Remote Sensing 138, pp. 139150

[15] Benediktsson, J.A., Palmason, J.A., Sveinsson, J.R., 2005. Classification of hyperspectral data from urban areas based on extended morphological profiles. IEEE Transactions on Geoscience and Remote Sensing 43(3), pp. 480-491.

[16] Dalla Mura, M., Benediktsson, J.A., and Bruzzone, L., 2010. Classification of Hyperspectral Images with Extend Attribute Profiles and Feature Extraction Techniques. IEEE Geosciences and Remote Sensing Symposium 2010, pp.76-79.

[17] Ghamisi, P., Souza, R., Benediktsson, J. A., Zhu, X., Rittner, L., and Lotufo, R.A., 2016. Extinction profiles for the classification of remote sensing data. IEEE Transactions on Geoscience and Remote Sensing 54(10), pp.5631-5645.

[18] Krizhevsky, A., Sutskever, I., and Hinton, G. E., 2012. Imagenet classification with deep convolutional neural networks. Advances in neural information processing systems, pp. 1097 1105 .

[19] Szegedy, C., et al., 2015. Going deeper with convolutions. In Proceedings of the IEEE conference on computer vision and pattern recognition, pp. 1-9.

[20] Girshick, R., Donahue, J., Darrell, T., and Malik, J., 2014. Rich feature hierarchies for accurate object detection and semantic segmentation In Proceedings of the IEEE conference on computer vision and pattern recognition, pp. 580-587.

[21] Bordes, A., Glorot, X., Weston, J., and Bengio, Y., 2012. Joint learning of words and meaning representations for opentext semantic parsing In Artificial Intelligence and Statistics, pp. $127-135$.

[22] Chen, Y., Lin, Z., Zhao, X., Wang, G., and Gu, Y., 2014. Deep learning-based classification of hyperspectral data. IEEE Journal of Selected Topics in Applied Earth Observation and Remote Sensing 7 (6), pp. 2094-2107.

[23] Makantasis, K., Karantzalos, K., Doulamis, A., and Doulamis, N., 2015. Deep Supervised Learning for Hyperspectral Data Classification through Convolutional Neural Networks. In Proceedings of IEEE International Geoscience and Remote Sensing Symposium, Milan, Italy, 2015, pp. 4959-4962.

[24] Huang, G., Liu, Z., Weinberger, K.Q., 2017. Densely Connected Convolutional Networks. In Proceedings of IEEE Conference on Pattern Recognition and Computer Vision (CVPR), College Park, MD, USA, 2017, pp. 1-9.
[25] Szegedy, C., Liu, W., Jia, Y., Sermanet, P., Reed, S., Anguelov, D., Erhan, D., Vanhoucke, V., Rabinovich, A., 2015. Going Deeper with Convolutions. In Proceedings of the IEEE Conference on Computer Vision and Pattern Recognition, CVPR 2015, Boston, MA, USA, pp. 1-9.

[26] He, K., Zhang, X., Ren, S., and Sun, J., 2016. Deep Residual Learning for Image Recognition. In Proceedings of IEEE Conference on Computer Vision and Pattern Recognition (CVPR 2016), Las Vegas, NV, USA, pp. 770-778.

[27] Chen, Y., Jiang, H., Li, C., Jia, X., and Ghamisi, P., 2016. Deep Feature Extraction and Classification of Hyperspectral Images Based on Convolutional Neural Networks. IEEE Transactions on Geoscience and Remote Sensing 54(10), pp. 6232-6251.

[28] Teffahi, H., Yao, H., Chaib, S., \& Belabid, N., 2019. A novel spectral-spatial classification technique for multispectral images using extended multi-attribute profiles and sparse autoencoder. Remote Sensing Letters 10(1), pp.30-38.

[29] Benediktsson, J.A., and Ghamisi, P., 2015. Spectral-Spatial Classification of Hyperspectral Remote Sensing Images. Artech House, Boston, MA, USA. ISBN: 9781608078127.

[30] Yang, G., Gewali, U. B., Ientilucci, E., Gartley, M., and Monteiro, S.T., 2018. Dual-Channel DenseNet for Hyperspectral Image Classification. In IEEE International Geoscience and Remote Sensing Symposium, pp. 2595-2598.

[31] Meng, Z., Li, L., Jiao, L., Feng, Z., Tang, X., and Liang, M., 2019. Fully Dense Multiscale Fusion Network for Hyperspectral Image Classification. Remote Sensing 11(22), p.2718.

[32] He, K., Zhang, X., Ren, S., and Sun, J., 2016. Identity mappings in deep residual networks. In Proceedings of the European Conference on Computer Vision (ECCV 2016), Amsterdam, The Netherlands, pp. 630-645.

[33] Loffe, S., and Szegedy, C., 2015. Batch normalization: Accelerating deep network training by reducing internal covariate shift. In Proceding of an International Conference of Machine Learning (ICML, Lile, France), 1, pp. 448-456.

[34] Bai, Y., Zhang, Q., Lu, Z., and Zhang, Y., 2019. SSDCDenseNet: A Cost-Effective End-to-End Spectral-Spatial DualChannel Dense Network for Hyperspectral Image Classification. IEEE Access, 7, pp. 84876-84889. 